\title{
Preface to the special issue
}

\author{
John Franco
}

Published online: 27 August 2011

(C) Springer Science+Business Media B.V. 2011

The series "International Symposium on Artificial Intelligence and Mathematics" is held every two years in Fort Lauderdale. While the general scope of the series is broad, and even includes the interaction of AI with Operations Research, each meeting typically includes a special focus on one or two particular topics. In the 2010 meeting, about half the papers were in the track Boolean and pseudo-Boolean functions which was organized by Endre Boros and Yves Crama, another four were in the track Constraint programming and hybrid methods organized by John Hooker, and the remaining papers covered a wide range of topics that tie AI to mathematics. This special issue of AMAI contains four papers from the third track and one from the first track, chosen by the organizers to be expanded and polished into journal versions by their excellence in written and oral presentation. Two papers are on Bayesian updating, one on the multi-armed bandit problem, one on concept analysis in data mining, and one on minimizing clausal data bases. In what follows, we try to set the context for each of these papers.

A Bayesian network is a probabilistic directed acyclic graph for modeling a set of random variables and their conditional dependencies, for example, between a set of diseases and symptoms. Bayesian networks may be used to generate explanations for given evidence, such as finding the most likely instantiation of a set of target variables given partial evidence on the remaining variables. Most Relevant Explanation (MRE) is the problem of generating an explanation that emphasizes the role of the most relevant target variables: if $e$ is a set of observed variable values and if $y_{1}, y_{2}, \ldots$ are all possible instantiations of all possible subsets of target variables, then $\mathrm{MRE}=\max _{i} \operatorname{Pr}\left(e \mid y_{i}\right) / \operatorname{Pr}\left(e \mid \neg y_{i}\right)$. In some respects, MRE estimates may be better than other Bayesian estimates. However, in the paper "Most Relevant Explanation: Computational complexity and approximation methods", Yuan, Lim, and Littman show that MRE is NP-hard. The authors then propose and evaluate

J. Franco $(\bowtie)$

University of Cincinnati, Cincinnati, USA

e-mail: franco@gauss.ececs.uc.edu 
several approximation algorithms for MRE. Empirical results indicate optimal MRE solutions can often be found using the approximation algorithms.

Jeffrey's rule may also be used to update explanations given evidence. Call a possible set of variable values a state and a set of states an event. Specify uncertain evidence as a mutually exhaustive and exclusive set of events $\left\{e_{1}, \ldots, e_{n}\right\}$. Before updating, suppose $P\left(e_{i}\right)$ is the probability that expresses the uncertainty of event $e_{i}$. According to Jeffrey's rule, the probabilities of every event $q$ must be updated so that $P\left(q \mid e_{i}\right)=P^{\prime}\left(q \mid e_{i}\right)$ for all $q$ and all $e_{i}$ where $P^{\prime}$ is the conditional probability of $q$ after updating. That is, the conditional probability of any event given any uncertain event does not change after updating. This restriction allows a straightforward application of Bayes' rule and marginalization to uniquely determine the updated probabilities. Jeffrey's rule has a counterpart in possibility theory. There, a function $\pi$ maps states to $[0,1]: \pi(s)=0$ means $s$ is impossible, $\pi(s)=1$ means $s$ is completely plausible. There are two variants to possibility theory: qualitative, where $\pi$ maps states to an ordinal scale where only the "ordering" of the values is important; and quantitative, where the possibilistic scale $[0,1]$ is numerical and possibility degrees are like numeric values that can be manipulated by arithmetic operators. In the paper "Jeffrey's rule of conditioning in a possibilistic framework: An analysis of the existence and uniqueness of the solution", Benferhat, Tabia and Sedki show that, in the quantitative possibilistic setting, solutions computed using possibilistic counterparts of Jeffrey's rule are unique; but, in the qualitative possibilistic setting, there might not be a solution that satisfies the conditions of Jeffrey's rule: namely, if there exists an uncertain event whose revision results in losing the relative plausibility order of its own elementary events.

Multi-armed bandit (MAB) problems are a class of sequential resource allocation problems concerned with allocating one or more resources among several alternative and competing processes. Obviously, this problem is encountered when large projects, institutions, or firms need to make decisions involving resource allocation to maximize some measure of profit. The problem may be modeled as a series of slot machines, each representing a process: when a lever is pulled on a machine, the associated process is given a resource. But, since information about each process is incomplete, the payoff resulting from allocating this resource may be a number that is obtained randomly from some distribution and may even be 0 . The problem is to find a sequence of lever pulls that maximizes (expected) payoff. This is clearly a statistics problem that sees application in Operations Research. It becomes a problem in Machine Intelligence when the decision procedure makes use of learned information to make the optimal next choice in lever pull. In the paper "Multi-armed bandits with episode context", Christopher Rosin refines the above model to include contextual side information that may be available at the start of the lever pulling episode and does not change during it. This model is presented as an alternative to prior models that have no initial context and those whose context may change on every lever pull. Rosin presents an efficient algorithm for solving this variant of MAB. It requires some sort of predictor to suggest an initial recommendation but can make use of information obtained during further exploration. Rosin shows that the algorithm can benefit greatly from an accurate predictor but can also recover during exploration from bad choices presented by an inaccurate predictor.

Replacing a CNF formula that represents a knowledge base, say for an expert system, with a logically equivalent CNF formula that consists of the minimum 
possible number of clauses can improve the performance of system query considerably. The problem of finding a minimum length equivalent formula is NPhard even for inputs that are Horn formulas. However, for some subclasses of Boolean functions the problem is solvable in polynomial time. These subclasses include quadratic, acyclic Horn, and quasi-acyclic Horn functions. A new class, called Component-wise Quadratic (CQ) Horn functions, is a generalization of the latter two classes and properly contains all three. It has been shown that the minimization problem is solved in polynomial time for any CQ Horn function $f$ given that the input is a CNF representation of $f$ where every clause is a prime implicate of $f$ and dropping any clause results in a formula that is not equivalent to $f$. In "Disjoint essential sets of implicates of a CQ Horn function" Cepek and Kucera show that, given a CQ Horn function $f$, the maximum number of pairwise disjoint essential sets of implicates of $f$ equals the minimum number of clauses in a CNF representation of $f$. So, quite likely, the properties of being polynomially minimizable and being coverable by essential sets are closely related. This leaves open the question whether CNF minimization is in the class $P$ for the set of all coverable functions, or is it just a coincidence that all known polynomially minimizable classes are inside the set of coverable functions?

Decision problems in areas such as data mining and social networking seek to identify and reason about large groups of entities over many possible attributes. Formal Concept Analysis (FCA) is a proposed method for facilitating such reasoning. In FCA one builds a $0-1$ matrix $M$, called a context, whose rows are indexed on entities, whose columns are indexed on attributes, and such that $M_{i, j}=1$ if and only if entity $i$ has attribute $j$. A concept is a submatrix of all $1 \mathrm{~s}$ after suitable row and column permutations. There are usually very many concepts in a context and these may be organized into a lattice structure. In Artificial Intelligence this knowledge structure would be called an ontology. Such a structure supports the ability to reason about concepts but this may be difficult if the number of concepts is high, which it usually is. Clustering and/or segmenting concepts may reduce this difficulty significantly. A concept similarity measure may be defined and used to do this. In "Similarity measures in Formal Concept Analysis", Alqadah and Bhatnagar proposed four similarity measures and test them on empirical data (see the paper for details). One of the measures was shown to provide superior results on sparse data.

John Franco, July 2011 